\title{
A telemedicine intervention to improve adherence to continuous positive airway pressure: a randomised controlled trial
}

\author{
David Sparrow, ${ }^{1,2}$ Mark Aloia, ${ }^{3}$ Deborah A DeMolles, ${ }^{1}$ Daniel J Gottlieb ${ }^{1,2}$
}

\section{See Editorial, p 1035}

- An additional appendix is published online only. To view this file please visit the journa online (http://thorax.bmj.com).

${ }^{1}$ VA Boston Healthcare System, Boston, Massachusetts, USA ${ }^{2}$ The Pulmonary Center, Boston University School of Medicine, Boston, Massachusetts, USA

${ }^{3}$ National Jewish Health, Denver, Colorado, USA

\section{Correspondence to} Dr David Sparrow, VA Boston Healthcare System (151N), 150 South Huntington Ave, Boston MA 02130, USA

david.sparrow@va.gov

Received 15 December 2009 Accepted 23 August 2010 Published Online First 29 September 2010
ABSTRAC

Background Continuous positive airway pressure (CPAP) is the most widely prescribed treatment for obstructive sleep apnoea syndrome (OSAS). Although it has been shown to improve the symptoms of OSAS, many patients have difficulty adhering to this treatment. The purpose of this study was to investigate the effectiveness of an automated telemedicine intervention to improve adherence to CPAP.

Methods A randomised clinical trial was undertaken in 250 patients being started on CPAP therapy for OSAS. Patients were randomly assigned to use a theory-driven interactive voice response system designed to improve CPAP adherence (telephone-linked communications for CPAP (TLC-CPAP), $n=124$ ) or to an attention placebo control $(n=126)$ for 12 months. TLC-CPAP monitors patients' self-reported behaviour and CPAP-related symptoms and provides feedback and counselling through a structured dialogue to enhance motivation to use CPAP. A Sleep Symptoms Checklist, the Functional Outcomes of Sleep Questionnaire, the Center for Epidemiological Studies Depression Scale and the Psychomotor Vigilance Task were administered at study entry and at 6-month and 12-month follow-up. Hours of CPAP usage at effective mask pressure were measured by the CPAP device stored in its memory and retrieved at each visit. Results Median observed CPAP use in patients randomised to TLC-CPAP was approximately $1 \mathrm{~h} /$ night higher than in the control subjects at 6 months and $2 \mathrm{~h} /$ night higher at 12 months. Using generalised estimating equation modelling, the intervention had a significant effect on CPAP adherence. For secondary analysis, the effect of CPAP adherence on the secondary outcomes was analysed. CPAP adherence was significantly associated with a greater reduction in sleep apnoea symptoms and depressive symptoms and a greater improvement in functional status. No significant association was observed between CPAP adherence and reaction time.

Conclusions The TLC-CPAP intervention resulted in improved CPAP adherence, which was associated with improved functional status and fewer depressive symptoms. Clinical trial.gov: NCT00232544.

\section{INTRODUCTION}

Obstructive sleep apnoea syndrome (OSAS), which occurs in at least $4 \%$ and $2 \%$ of middle-age men and women, respectively, in the USA, ${ }^{1}$ contributes to neurobehavioral morbidity hypertension, ${ }^{2-5}$ cardiovascular disease $^{67}$ and all-cause mortality. ${ }^{89}$ The initial treatment for most patients with OSAS is nasal continuous positive airway pressure
(CPAP). CPAP eliminates apnoea ${ }^{10} 11$ and improves daytime sleepiness and quality of life (QOL). ${ }^{11-13}$

Patient adherence to the prescribed use of CPAP is poor; $20-33 \%$ of patients discontinue CPAP use within 4 months and use among those who do not is less than optimal. ${ }^{14-17}$ Patients discontinue or underuse CPAP therapy due to a myriad of side effects $^{151819}$ and a lack of knowledge on how to ameliorate them. Other causes for non-adherence are related to theoretical models of how individuals go about making health-related behaviour changes. Psychological theories such as social cognitive theory ${ }^{20}$ suggest that individuals make changes to their behavioural patterns based on the relative weighing of the pros and cons of making changes (referred to as decisional balance), and on their perceived confidence that they can make a behaviour change in times when such a change is expected to be difficult (referred to as self-efficacy). These factors are explained more completely and applied to CPAP use elsewhere. ${ }^{21}$ Although not specific to CPAP use, the factors have been shown strongly to predict non-adherence to CPAP, making them targets for intervention. ${ }^{22}$

Patient adherence during the first days after initiation of CPAP therapy is a powerful predictor of long-term adherence. ${ }^{14} 1623$ Thus, to enhance long-term adherence, it is crucial to improve patients' understanding of the expected benefits of CPAP use, increase motivation to use this therapy, recognise and address side effects, and monitor and promote adherence to CPAP therapy during the initial period of use. Several small randomised controlled trials (RCTs) have evaluated a variety of education, support and behavioural interventions to increase CPAP utilisation over periods as long as 6 months. Two small studies found that weekly human telephone support over a 2-3 month period in new or continuing CPAP users resulted in a $1.0-1.3 \mathrm{~h} /$ night higher utilisation compared with a usual care group. ${ }^{24}$ Hoy et al randomised a larger sample of 80 patients with newly diagnosed OSAS to receive either standard or intensive support, the latter including home CPAP education, two additional nights of CPAP titration to resolve CPAP problems and home visits at 1, 2 and 4 weeks and at 4 months. ${ }^{26}$ This intensive support resulted in a $1.6 \mathrm{~h} /$ night greater CPAP utilisation at 6 months. Hui et al, ${ }^{27}$ in contrast, found no increase in CPAP utilisation after 12 weeks following an augmented support protocol including a $15 \mathrm{~min}$ educational video, an additional 15 min educational session and six calls from a respiratory nurse during the first 4 weeks and every 4 weeks thereafter, although the 
average utilisation of $5.3 \mathrm{~h} /$ night in the usual care group was similar to that of the intensive care groups in the other studies cited above. ${ }^{24-26}$ One previous study of a face-to-face motivational intervention for CPAP use did demonstrate efficacy at reducing CPAP discontinuance rates by approximately $25 \% .{ }^{28}$ More recently, an RCT has evaluated the use of a cognitive behavioural therapy intervention to enhance CPAP utilisation. ${ }^{29}$ Compared with usual care, this intervention-consisting of two $1 \mathrm{~h}$ sessions, a 15 min video and written materials, all provided in advance of CPAP titration-resulted in a much higher proportion of patients using CPAP at least $4 \mathrm{~h} /$ night after 28 days (77\% vs $31 \%)$.

Telephone-linked communications (TLC) systems offer an effective low-cost and convenient way of providing information, advice and counselling to improve patient adherence. ${ }^{30}$ Previous studies have demonstrated the applicability of TLC technology in the care of adults with a wide variety of chronic health conditions (eg, hypertension, chronic obstructive pulmonary disease $)^{31} 32$ and its efficacy in modifying health behaviour (medication-taking, diet and exercise). ${ }^{30} 3133$ TLC interventions can also be tailored to follow the tenets of theories of behaviour change such as the social cognitive theory. We developed a new TLC system for promoting adherence to CPAP (TLC-CPAP) as an automated telephone-based and low-cost alternative to prior personnel intensive interventions ${ }^{24} 262729$ and examined its impact in an RCT.

\section{METHODS \\ Study sample}

Participants were patients undergoing initial set-up of fixed pressure CPAP or BiPAP at one of two Boston area medical centres: the VA Boston Healthcare System (VABHS) and the Boston Medical Center (BMC). Those aged 18-80 years with a physician diagnosis of OSAS and with polysomnography demonstrating an apnoea-hypopnoea index (AHI) $>10$ were invited to participate. We went to the homes of interested patients prior to the initiation of CPAP therapy. After informed consent was obtained, participants completed a baseline assessment and received an OSAS educational session that included a simple description of the physiology of OSAS, common OSAS symptoms and potential health risks and discussion of the participant's own OSAS severity and response to CPAP therapy. Participants were then randomised to one of two groups: one group used an educational control TLC system ('attention placebo control group') and the other group used the TLC-CPAP system. Randomisation was stratified by sex, age and AHI using a randomised block design to ensure balance of these factors in the treatment arms.

\section{TLC-CPAP intervention}

The TLC-CPAP was designed around the concepts of motivational interviewing, ${ }^{34}$ a patient-centred approach to increase motivation to engage in a health behaviour by addressing the themes of perceived importance of using CPAP and confidence to adhere to CPAP. The TLC-CPAP system was automated and adapted from the intervention described by Aloia et $a l^{21}$ as Motivational Enhancement for CPAP. TLC-CPAP uses digitised human speech to speak to the patients and the patients communicate via the touch-tone keypad of their telephones. The TLC-CPAP content includes assessment of the patient's perceptions about and experiences with OSAS and CPAP therapy and the patient's reported CPAP use (hours per night and nights per week) during the week preceding each call; assessment of the patient's goals with regard to OSAS therapy; and feedback and counselling to enhance motivation to use CPAP and address barriers and poor self-efficacy. While side effect management was not the focus of the TLC-CPAP intervention, a side effect management module addressing mucocutaneous side effects, air leaks and mask discomfort was developed and incorporated in the dialogues as appropriate.

At the beginning of a TLC-CPAP call the user was instructed to enter a personal password to ensure security and confidentiality. Each call began with an assessment of the self-reported frequency and duration of CPAP usage during the previous week, followed by one of several motivational counselling modules. An outline of the content of each call is shown in appendix $A$ in the online supplement; more detail of this content can be found in Aloia et al, ${ }^{21}$ from which the calls were adapted. Confidence and decisional balance were assessed individually by the TLC-CPAP system. Barriers and facilitators to using CPAP nightly were probed to highlight the discrepancies between why patients might seek treatment and what was keeping them from using CPAP. When the cons of using treatment were higher than the pros or confidence was low, the system assessed if the participant was having any side effects with CPAP and ascertained the severity of OSAS-related symptoms including snoring, breathing pauses and daytime sleepiness. If the participant reported excessive side effects or OSAS symptoms, the system then recommended that $\mathrm{s} /$ he contact his or her physician to discuss the problems. Other concepts incorporated in the calls included information exchange, an assessment of what the participant values with regard to his/her treatment and overall health, goal setting and reminders of what was previously discussed in previous calls. Information exchange included topics such as: (1) comparisons between reaction times with untreated OSAS and those under the influence of alcohol; (2) the medical comorbidities common to OSAS; and (3) the risks of having a motor vehicle accident with untreated OSAS. Personalised information was elicited from participants including perception of problems before CPAP, perceived benefits from treatment and personal goals for CPAP use.

Participants were required to make weekly calls to TLC-CPAP during the first month beginning 3 days after starting CPAP therapy and thereafter monthly for the 12-month duration of the study. The computer system called the participants if they did not make a call at the expected times (some grace time was allowed).

Routine printed reports were sent to the participants' physicians biweekly during the first month and monthly thereafter. These reports included information on the frequency and duration of CPAP use, side effects and OSAS symptoms. In addition, reports were sent during unscheduled weeks of the first month when a participant reported underuse of CPAP (for the 3-day call, $<6$ h total over the 3 days; for a weekly call, $<4 \mathrm{~h} /$ night on nights the CPAP was used) or side effects including mask discomfort, dryness of nose and throat, claustrophobia and belching or air swallowing, deemed by the participant to be of moderate to severe intensity. Notification was sent to the physician if a participant reported discontinuing CPAP at the physician's instruction to confirm that instruction.

\section{Attention placebo control}

Participants randomised to the attention placebo control group received general health education via a TLC system. This system provides general information about a variety of health topics via telephone calls delivered on the same schedule as the TLC-CPAP calls made by the intervention group. At each call, participants selected a topic from a list of 61 content areas that included 
common symptoms, medical conditions and preventive medicine topics. The health information dialogues were adapted from Harvard Health Letter articles (http://www.health.harvard.edu). They were developed to allow users to identify subtopics about which they wanted more information and to skip others, and avoided long stretches of uninterrupted talking by the system. ${ }^{35}$

\section{Data collection}

Data were collected during three home visits 6 months apart. At the baseline examination, height and weight were measured and subjects completed the Sleep Symptoms Checklist, the Functional Outcomes of Sleep Questionnaire (FOSQ), the Center for Epidemiological Studies Depression (CES-D) Scale, the Psychomotor Vigilance Task (PVT), the CPAP Self-Efficacy Index and the CPAP Decisional Balance Index. At the 6-month and 12-month visits these measures were repeated and data on CPAP use time were retrieved. CPAP use time was defined as the average hours per night of CPAP usage at effective mask pressure (defined as pressure within $2 \mathrm{~cm} \mathrm{H}_{2} \mathrm{O}$ of the prescribed pressure). ${ }^{14} 17$ These data were either downloaded from the memory card of the CPAP device or obtained by direct interrogation of CPAP devices that did not have memory cards. All data were collected by research assistants blind to group assignment.

The FOSO is a self-reported measure designed to assess the impact of disorders of excessive sleepiness on multiple activities of daily living. ${ }^{36}$ The Sleep Symptoms Checklist ${ }^{26}$ measures the frequency of nine sleep-related symptoms using a 6-point Likert scale (ranging from never $=0$ to always $=5$ ); a score is calculated by adding the responses for the nine items. Behavioural alertness was assessed using the PVT. ${ }^{37}$ Participants were instructed to respond to the appearance of a visual stimulus by pushing a response button as quickly as possible. Reaction times were collected and the mean reaction time and the mean reciprocal of the $10 \%$ slowest reaction times were extracted using a software program. $^{37}$

To assess whether the TLC-CPAP intervention influenced attitudes towards CPAP treatment (eg, motivation to use CPAP) we administered two scales developed by Stepnowsky and colleagues. $^{22}$ The CPAP Self-Efficacy Index was constructed using statements to assess the extent to which the patients believe that they can follow through with CPAP treatment. The CPAP Decisional Balance index was designed for assessing decision-making processes in using CPAP. It consists of both pro items (which assess the benefits of using CPAP) and con items (which assess the costs to the patient of using CPAP). Together, these indices provide evidence for changes in the actual motivation of participants to use CPAP and can be considered potential mechanisms of change in this study.

\section{Data analysis}

Analyses were performed by intention to treat. Data were summarised as medians and interquartile ranges (IOR). Log-transformation was performed on the outcomes to help normalise the data. However, to avoid taking the log of 0 we added a small constant (0.1) to all values of mean hours of CPAP use. The primary analysis took the form

$$
\begin{aligned}
\ln (\text { mean hours CPAP use })= & a+b \cdot \text { INT }+c \cdot \text { TIME }+d \cdot \text { SEX } \\
& +e \cdot \text { AHI }+\mathrm{f} \cdot \text { AGE }
\end{aligned}
$$

where INT is a dummy variable indicating that the subject is $(\mathrm{INT}=1)$ or is not $(\mathrm{INT}=0)$ receiving the TLC-CPAP intervention; TIME is a dummy variable indicating whether the outcome is recorded at $6(\mathrm{TIME}=0)$ or $12(\mathrm{TIME}=1)$ months post-baseline; and sex, AHI and age are the baseline covariates.
Because each individual contributes two values (at 6 months and 12 months), generalised estimating equations (GEE) ${ }^{38}$ were used to estimate the effects properly adjusted for non-independent contributions. Validity of large sample inference with GEE does not require normality of the response. ${ }^{39}$ The exchangeable working covariance model was used as there is only one intrasubject correlation parameter for the two-period study, along with the identity link function and the normal distribution. To account for missing data in the outcome of CPAP use due to loss to follow-up, we implemented a multiple imputation procedure. Five datasets were generated with plausible values imputed for the missing values. Each imputed dataset was then analysed using GEE and the resulting parameter estimates were appropriately combined across datasets to yield final estimates. Analyses were performed using SAS Version 9.1.

\section{RESULTS}

A total of 250 participants were randomised to the TLC-CPAP $(n=124)$ or attention control $(n=126)$ groups. The median age of the participants was 55.0 years (IOR 46.0-63.0), the median body mass index was $35.1 \mathrm{~kg} / \mathrm{m}^{2}$ (IOR $31.0-41.3$ ) and $82 \%$ were men. The AHI range was 10-154 events/h, with $60 \%$ having $\mathrm{AHI} \geq 30$. The baseline characteristics of the intervention and control groups were similar (table 1). CPAP adherence data were available from either the 6- or 12-month follow-up visit in $93.6 \%$ of subjects (figure 1), who were therefore included in the primary analysis.

The median observed CPAP use in patients randomised to TLC-CPAP was $2.40 \mathrm{~h} /$ night and $2.98 \mathrm{~h} /$ night at 6 months and 12 months, respectively; in control subjects the values were $1.48 \mathrm{~h} /$ night and $0.99 \mathrm{~h} /$ night. Using a commonly accepted definition of adherence ( $>4 \mathrm{~h} /$ night) by the end of 12 months, the TLC-CPAP intervention was associated with a $30 \%$ higher rate of CPAP use $(44.7 \%$ of intervention group using CPAP $4 \mathrm{~h} /$ night vs $34.5 \%$ of control group). Using GEE modelling, we found a significant intervention effect in the complete case $(\mathrm{n}=234)$ analysis $(1.70 \mathrm{~h} /$ night; $95 \%$ CI 1.17 to $2.47 ; \mathrm{p}=0.006)$. The intervention effect remained significant in the multiple imputation GEE analysis (1.71 h/night; 95\% CI 1.18 to 2.48; $\mathrm{p}=0.004$, figure 2). Although the magnitude of the benefit was approximately twice as great at 12 months compared with 6 months, a formal test of time by intervention interaction was performed by adding a time $X$ intervention interaction term to the GEE analysis. This interaction was not statistically significant.

Table 1 Baseline characteristics of study subjects

\begin{tabular}{lcc}
\hline & \multicolumn{2}{c}{ Intervention (TLC-CPAP) } \\
\hline Age (years) & $56.0(48.0-63.0)$ & $54.0(45.0-62.0)$ \\
Male $(\%)$ & $100(80.7 \%)$ & $105(83.3 \%)$ \\
Body mass index $\left(\mathrm{kg} / \mathrm{m}^{2}\right)$ & $34.4(30.1-40.1)$ & $35.9(31.9-42.1)$ \\
Apnoea-hypopnoea index (events/h) & $36.0(22.0-63.0)$ & $40.5(21.0-64.0)$ \\
Epworth Sleepiness Scale & $10.0(6.0-15.0)$ & $11.0(8.0-15.0)$ \\
FOSO & $16.6(13.5-18.2)$ & $16.1(13.6-18.3)$ \\
Sleep Symptoms Checklist & $30.0(24.0-36.0)$ & $31.0(26.0-37.0)$ \\
CES-D & $18.0(14.0-26.0)$ & $22.0(15.0-31.0)$
\end{tabular}

Visual reaction time

Mean reaction time (ms) $303.3(266.5-361.6) \quad 308.0(283.2-357.9)$

Mean slowest reaction time (ms) 505.9 (417.0-668.3) 534.2 (460.4-719.0)

All results are presented as median (IQR) values. There were no differences between the two groups on these characteristics by Mann-Whitney $U$ test at $p<0.05$.

CES-D, Center for Epidemiological Studies Depression (CES-D) Scale; CPAP, continuous positive airway pressure; FOSQ, Functional Outcomes of Sleep Questionnaire; TLC, telephone-linked communication. 


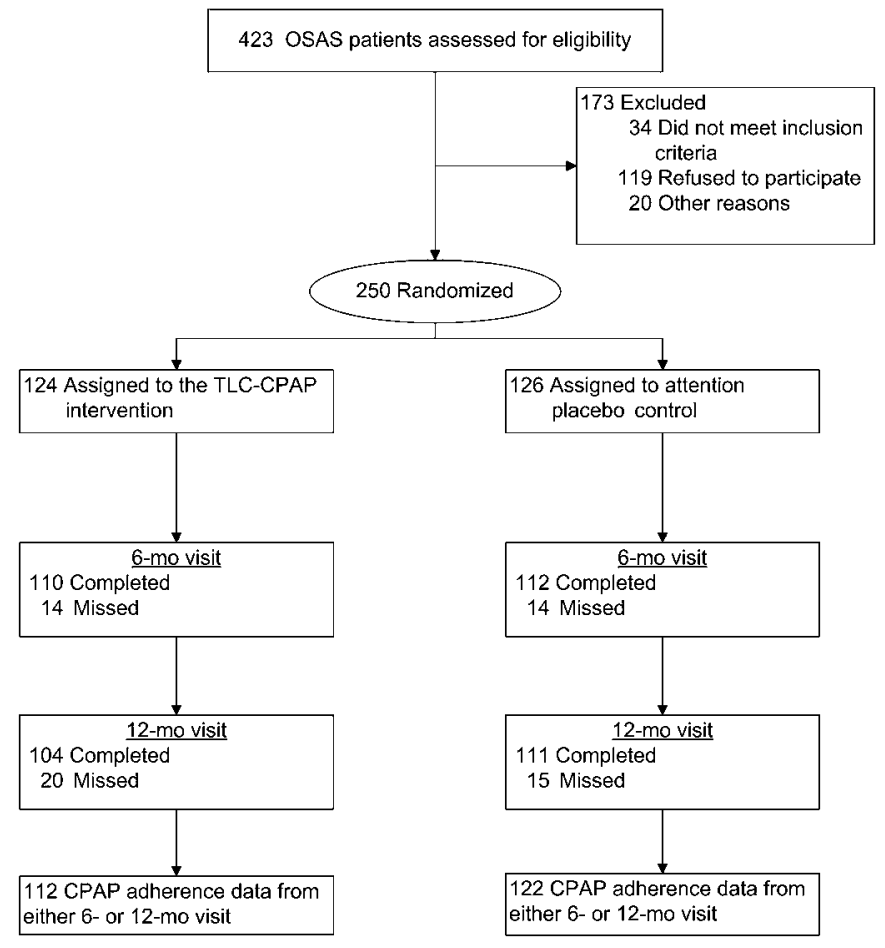

Figure 1 Participant flow. CPAP, continuous positive airway pressure; OSAS, obstructive sleep apnoea syndrome; TLC, telephone-linked communication.

We also examined the relationship between the amount of CPAP use and disease-specific QOL and OSAS symptoms (table 2). The amount of CPAP use in the previous 6 months was predictive of improvements in QOL (FOSQ), sleep symptoms and depression (CES-D). The amount of CPAP use did not influence daytime vigilance as assessed by reaction time measurements.

Mediation analysis was performed to examine whether the effect of TLC-CPAP on CPAP adherence was mediated by changes in motivation (eg, self-efficacy and decisional balance as measured by the CPAP Self-Efficacy and Decisional Balance Indices). The intervention and control groups were similar at baseline on both constructs (table 3). At 6 and 12 months the intervention group scored significantly higher than the control group on both measures. Moreover, each of these measures was a significant predictor of both concurrent and future adherence to CPAP. When these measures were included in multivariable models predicting CPAP adherence, the estimated effect of the

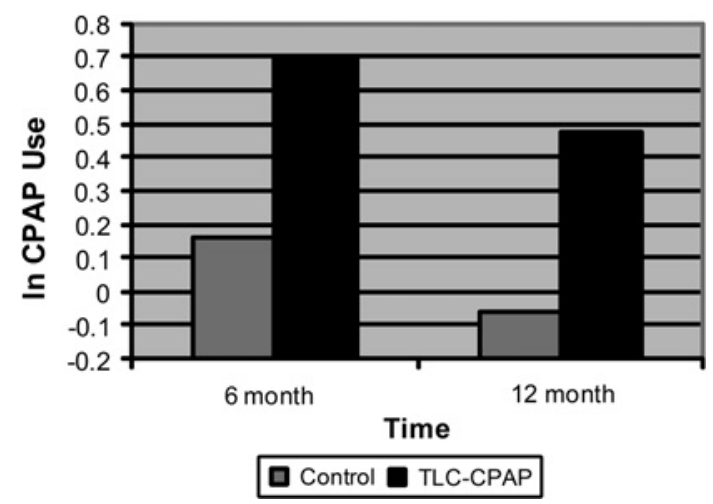

Figure 2 Use of continuous positive airway pressure (CPAP) by group over time. TLC, telephone-linked communication.
Table 2 Association of amount of CPAP use in the previous 6 months with disease-specific quality of life, sleep symptoms, depression and daytime vigilance using generalised estimating equations

\begin{tabular}{|c|c|c|c|c|}
\hline Outcome variable & $\begin{array}{l}\text { Regression } \\
\text { coefficient }\end{array}$ & SE & $95 \% \mathrm{CI}$ & $\begin{array}{l}p \\
\text { Value }\end{array}$ \\
\hline FOSO & 0.021 & 0.007 & 0.008 to 0.035 & 0.003 \\
\hline Sleep Symptoms Checklist & -0.070 & 0.013 & -0.096 to -0.045 & $<0.001$ \\
\hline CES-D & -0.028 & 0.014 & -0.056 to -0.000 & 0.048 \\
\hline
\end{tabular}

Visual reaction time

Mean reaction time (ms) $\quad-0.007 \quad 0.008-0.023$ to $0.008 \quad 0.369$

$\begin{array}{llll}\text { Mean slowest reaction time (ms) }-0.010 & 0.014 & -0.037 \text { to } 0.017 & 0.457\end{array}$

CES-D, Center for Epidemiological Studies Depression (CES-D) Scale; CPAP, continuous positive airway pressure; FOSQ, Functional Outcomes of Sleep Questionnaire.

TLC-CPAP intervention decreased by $60 \%$, suggesting substantial mediation of the intervention effect by changes in self-efficacy and decisional balance.

\section{DISCUSSION}

In this large RCT of 250 patients with newly diagnosed OSA we found that a 12-month telemedicine intervention resulted in a median CPAP usage that was $0.9 \mathrm{~h} /$ night higher than that of an attention control group after 6 months, and $2.0 \mathrm{~h} /$ night higher after 12 months. Previous studies have demonstrated some efficacy of educational, behavioural and/or theory-driven interventions for improving adherence to positive airway pressure. $^{24-29}$ None of these trials, however, offered the benefits of a TLC system, which is low-cost, easy to deliver and a potentially powerful first step in an attempt to improve adherence among larger samples of patients. The focus on theory was not lost in the translation of the motivational intervention into this TLC approach. These data suggest that the theoretical background for the intervention was the key mechanism of action for the improvement in adherence; changes in decisional balance and self-efficacy mediated the group-related changes in adherence, suggesting that the intervention had its desired effect on these important social cognitive constructs despite being delivered over the telephone.

The magnitude of the adherence effect seen in this study was similar to that observed with other more labour-intensive approaches $^{24-26}$ over a 6-month period, and this effect was maintained over a 12-month period. Although the effect was similar to that reported from other interventions, the median adherence was low relative to these previous studies, reflecting a high proportion of subjects (19\%) not using CPAP at all. In this regard, the study by Richards et al is of particular interest as the increase in CPAP adherence of that intervention, delivered prior to CPAP titration, appears to reflect primarily a reduction in the proportion of patients refusing CPAP titration from $41 \%$ in the usual care group to $8 \%$ in the intervention group. ${ }^{29}$ As our

Table 3 Levels of continuous positive airway pressure (CPAP) Self-Efficacy Index and CPAP Decisional Balance Index over time

\begin{tabular}{|c|c|c|c|c|c|c|}
\hline & \multicolumn{2}{|l|}{ Baseline } & \multicolumn{2}{|c|}{6 months } & \multicolumn{2}{|c|}{12 months } \\
\hline & Median & IOR & Median & IOR & Median & IOR \\
\hline \multicolumn{7}{|c|}{ CPAP Self-Efficacy Index } \\
\hline Intervention & 4.2 & $3.6-4.8$ & 4.4 & $3.8-5.0$ & 4.6 & $3.6-5.0$ \\
\hline Control & 4.2 & $3.6-4.8$ & 4.2 & $3.0-4.8$ & 4.2 & $3.0-5.0$ \\
\hline \multicolumn{7}{|c|}{ CPAP Decisional Balance Index } \\
\hline Intervention & -4.0 & $-8.0-4.0$ & 1.0 & $-6.0-7.0$ & 0.0 & $-6.0-6.0$ \\
\hline Control & -3.0 & $-9.0-4.0$ & -3.0 & $-10.5-4.0$ & -2.0 & $-11.0-4.0$ \\
\hline
\end{tabular}


telemedicine intervention did not begin until after CPAP was set up in the home, it may have been delivered too late to influence initial acceptance of CPAP. This may be a particularly important factor in this patient care setting where, owing to a relative scarcity of sleep specialists, most patients undergo laboratory polysomnography and CPAP titration in advance of a face-toface evaluation by a sleep specialist. Typically, CPAP is ordered for these patients and delivered to the home by a durable medical equipment company respiratory therapist with subsequent clinical follow-up by a sleep specialist to the assess response to therapy. This institution of CPAP treatment without prior discussion of the diagnosis and treatment options with a sleep specialist may have contributed to a low CPAP acceptance rate.

We found that greater adherence to CPAP was associated with a greater reduction in sleep apnoea symptoms such as snoring, breathing pauses, non-refreshing sleep and drowsy driving. Greater CPAP adherence was also associated with a greater improvement in functional status as measured by the FOSO. Weaver et al have reported a linear increase in the FOSO score with increasing CPAP adherence up to adherence levels of $7 \mathrm{~h} /$ night. ${ }^{40}$ It has been reported that CPAP use is associated with both short- and long-term decreases in depressive symptoms. ${ }^{41}$ In this study we found that greater CPAP adherence was associated with a significantly greater reduction in depression symptoms on the CES-D. In contrast to a previous study showing that CPAP adherence was associated with a greater improvement in reaction time using the Conners' Continuous Performance Test $^{42}$ we found no significant relationship between CPAP adherence and visual reaction time on the PVT, although differences were in the expected direction. Overall, these data are consistent with the literature suggesting that greater adherence to CPAP is associated with greater improvement in functional status, depressive symptoms and vigilance.

There are some important limitations to this study. First, adherence among all of our participants was lower than that of other published studies. As discussed above, this may reflect the fact that individuals received their intervention after CPAP titration, suggesting that we may have already lost individuals who had a negative titration experience. It is also possible that individuals who thought they would need help with adhering to CPAP were more likely to enrol in this study. Only replication can address this possibility. Although the intervention was delivered over the telephone, participants also received in-person visits to collect data at 6-month intervals. While participant contact has been associated with improved health behaviours independent of a specific intervention (the 'Hawthorne effect'), the frequency of these visits is sufficiently low that they are unlikely to have had a major impact on behaviour and would, in any case, bias towards a null result.

In summary, this study demonstrates initially promising results of a motivational intervention, delivered over the telephone, to improve adherence to CPAP treatment. Additional interventions aimed at motivating CPAP adherence prior to set-up of CPAP should be considered in future studies.

Acknowledgements We thank Vincent Carey for his assistance in many aspects of the study design and data analysis.

Funding Department of Veterans Affairs, Veterans Health Administration, Health Services Research and Development Service. The views expressed in this article are those of the authors and do not necessarily represent the views of the Department of Veterans Affairs.

Competing interests MA is a paid employee of Philips/Respironics Inc and is a stockholder of Philips stock.
Ethics approval This study was conducted with the approval of the institutional review boards of the VA Boston Healthcare System and the Boston Medical Center and patients gave informed consent.

Provenance and peer review Not commissioned; externally peer reviewed.

\section{REFERENCES}

1. Young T, Palta M, Dempsey J, et al. The occurrence of sleep-disordered breathing among middle-aged adults. N Engl J Med 1993;328:1230-5.

2. Nieto FJ, Young TB, Lind BK, et al. Association of sleep-disordered breathing, sleep apnea, and hypertension in a large community-based study. JAMA 2000;283:1829-36

3. Peppard PE, Young T, Palta M, et al. Prospective study of the association between sleep-disordered breathing and hypertension. N Engl J Med 2000:342:1378-84.

4. Hla KM, Young TB, Bidwell T, et al. Sleep apnea and hypertension: a population-based study. Ann Intern Med 1994;120:382-8.

5. Stradling JR, Crosby JH. Relation between systemic hypertension and sleep hypoxemia or snoring: analysis of 748 men drawn from general practice. BMJ 1990:300:75-8.

6. Waller PC, Bhopal RS. Is snoring a cause of vascular disease? An epidemiologic review. Lancet 1989;1:143-6.

7. Hung J, Whitford EG, Parson RW, et al. Association of sleep apnea with myocardial infarction in men. Lancet 1990;336:261-4.

8. Partinen M, Jamieson A, Guilleminault C. Long-term outcome for obstructive sleep apnea syndrome patients: mortality. Chest 1988:94:1200-4.

9. He J, Kryger MH, Zorick FJ, et al. Mortality and apnea index in obstructive sleep apnea: experience in 385 male patients. Chest 1988;94:9-14.

10. Sullivan CE, Issa FG, Berthon-Jones M, et al. Reversal of obstructive sleep apnoea by continuous positive airway pressure applied through the nares. Lancet 1981;:1:862-5.

11. Rajagopal KR, Bennett LL, Dillard TA, et al. Overnight nasal CPAP improves hypersomnolence in sleep apnea. Chest 1986;90:172-6.

12. Engleman HM, Martin SE, Kingshott RN, et al. Randomized, placebo controlled tria of daytime function after continuous positive airway pressure (CPAP) therapy for the sleep apnoea /hypopnoea syndrome. Thorax 1998;53:341-5

13. Engleman HM, Kingshott RN, Wraith PK, et al. Randomized placebo-controlled crossover trial of continuous positive airway pressure for mild sleep apnea/hypopnea syndrome. Am J Respir Crit Care Med 1999;159:461-7.

14. Reeves-Hoche MK, Meck R, Zwillich CW. Nasal CPAP: an objective evaluation of patient compliance. Am J Respir Crit Care Med 1994;149:149-54.

15. Rolfe I, Olson LG, Saunders NA. Long-term acceptance of continuous positive airway pressure in obstructive sleep apnea. Am Rev Respir Dis 1991;144:1130-3.

16. Kribbs NB, Pack Al, Kline LR, et al. Objective measurement of patterns of nasal CPAP use by patients with obstructive sleep apnea. Am Rev Respir Dis 1993;147:887-95.

17. Engleman HM, Martin SE, Douglas NJ. Compliance with CPAP therapy in patients with the sleep apnoea/hypopnoea syndrome. Thorax 1994:49:263-6.

18. Hoffstein V, Viner S, Mateika S, et al. Treatment of obstructive sleep apnea with nasal continuous positive airway pressure. Am Rev Respir Dis 1992;145:841-5.

19. Waldhorn RF, Herrick TW, Nguyen MC, et al. Long-term compliance with nasal continuous positive airway pressure therapy of obstructive sleep apnea. Chest 1990:97:33-8

20. Bandura A. Social foundations of thought and action. A social cognitive theory. Englewood Cliffs, NJ: Prentice Hall, 1986.

21. Aloia MS, Arnedt JT, Riggs RL, et al. Clinical management of poor adherence to CPAP: motivational enhancement. Behav Sleep Med 2004;2:205-22.

22. Stepnowsky CJ Jr, Marler MR, Ancoli-Israel S. Determinants of nasal CPAP compliance. Sleep Med 2002:3:239-47.

23. Weaver TE, Kribbs NB, Pack Al, et al. Night-to-night variability in CPAP use over the first three months of treatment. Sleep 1997;20:278-83.

24. Chervin RD, Theut $\mathrm{S}$, Bassetti $\mathrm{C}$, et al. Compliance with nasal CPAP can be improved by simple interventions. Sleep 1997;20:278-83

25. Fletcher EC, Luckett RA. The effect of positive reinforcement on hourly compliance in nasal continuous positive airway pressure users with obstructive sleep apnea. Am Rev Respir Dis 1991;143:936-41.

26. Hoy CJ, Vennelle M, Kingshott RN, et al. Can intensive support improve continuous positive airway pressure use in patients with the sleep apnea/hypopnea syndrome? Am J Respir Crit Care Med 1999;159:1096-100.

27. Hui DSC, Chan JKW, Choy DKL, et al. Effects of augmented continuous positive airway pressure education and support on compliance and outcome in a Chinese population. Chest 2000:117:1410-16.

28. Aloia MS, Smith K, Arnedt JT, et al. Brief behavioral therapies reduce early PAP discontinuation rates in SAS: preliminary findings. Behav Sleep Med 2007:5:89-104

29. Richards D, Bartlett DJ, Wong K, et al. Increased adherence to CPAP with a group cognitive behavioral treatment intervention: a randomized trial. Sleep 2007:30:635-40

30. Friedman RH. Automated telephone conversations to assess health behavior and deliver behavioral interventions. J Med Syst 1998;22:95-102.

31. Friedman $\mathbf{R H}$, Kazis LE, Jette $A$, et al. A telecommunications system for monitoring and counseling patients with hypertension: impact on medication adherence and blood pressure control. Am J Hypertens 1996;9:2852-92. 
32. Young M, Sparrow D, Gottlieb D, et al. A telephone-linked computer system for COPD care. Chest 2001;119:1565-75.

33. Delichatsios HK, Friedman $\mathrm{RH}$, Glanz $\mathrm{K}$, et al. Randomized trial of a "talking computer" to improve adults' eating habits. Am J Health Promot 2001;15:215-24.

34. Miller W, Rollnick S. Motivational interviewing: preparing people for change. 2nd edn. New York: Guilford, 2002

35. Ramelson HZ, Bassey B, Friedman RH. The use of computer telephony to provide interactive health information. AMIA Annu Symp Proc 2003:539-43.

36. Weaver TE, Laizner AM, Evans LK, et al. An instrument to measure functional status outcomes for disorders of excessive sleepiness. Sleep 1997;20:835-43.

37. Dinges DF, Powell JW. Microcomputer analyses of performance on a portable simple visual RT task during sustained operations. Behav Res Methods Instrum Comput 1985;17:652-5.
38. Liang K-Y, Zeger SL. Longitudinal data analysis using generalized linear models. Biometrika 1986;73:13-22.

39. McCullagh P, Nelder JA. Generalized linear models. London: Chapman and Hall, 1989.

40. Weaver TE, Maislin G, Dinges DF, et al. Relationship between hours of CPAP use and achieving normal levels of sleepiness and daily functioning. Sleep 2007:30:711-19.

41. Schwartz DJ, Karatinos G. For individuals with obstructive sleep apnea, institution of CPAP therapy is associated with an amelioraton of symptoms of depression which is sustained long term. J Clin Sleep Med 2007;3:631-5.

42. Aloia MS, Iniczky N, Di Dio P, et al. Neuropsychological changes and treatment compliance in older adults with sleep apnea. J Psychosom Res 2003; $54: 71-6$

\section{Pulmonary puzzle}

\section{Eosinophilia, meningitis and pulmonary nodules in a young woman}

A 22-year-old woman was admitted unwell with headache, fever and encephalopathy. She was born in the UK to parents from Hong Kong. She had a past history of asthma, perennial rhinitis and atopic dermatitis for which she took a budesonide $100 \mu \mathrm{g}$ turbohaler and beclomethasone nasal spray. Three weeks before admission she had returned from holiday in Valencia and shortly after her return developed a 'flu-like illness followed by worsening asthma symptoms. Despite increasing usage of her corticosteroid turbohaler to eight puffs a day, her cough and wheeze worsened. Her GP prescribed a 5-day course of prednisolone $50 \mathrm{mg}$ daily (approximately $1 \mathrm{mg} / \mathrm{kg}$ ). Her asthma symptoms improved but she developed a headache 2 days after finishing the corticosteroid course. Over the next 5 days she became more unwell with worsening headache and presented to hospital.

On admission she was drowsy with signs of meningism. MRI brain showed a few small hyperintense foci bilaterally. Blood tests showed an eosinophil count of $16.8 \times 10^{9} / 1$ (total white cell count $\left.24.4 \times 10^{9} / 1\right)$ and IgE level of $5553 \mathrm{kU} / 1$. Lumbar puncture opening pressure was over $40 \mathrm{~cm}$ of water. Cerebrospinal fluid (CSF) white cell count was $33 / \mathrm{mm}^{3}$, with numerous lymphocytes and eosinophils. Serology for HIV-1 and 2 antibodies was negative and HIV RNA was undetectable. Testing for anti-neutrophil cytoplasmic antibodies and anti-nuclear antibodies was negative. A whole body CT scan showed multiple, small, randomly distributed pulmonary nodules (figure 1) and small volume lymphadenopathy in the chest.

\section{QUESTIONS}

What is the differential diagnosis of the nodular lesions on CT? What is the next investigation?

See page 1085 for answers

Paul E Pfeffer, ${ }^{1}$ Arjune Sen, ${ }^{2}$ Satya Das, ${ }^{3}$ Michael Sheaff, Anand Sivaramakrishnan, ${ }^{5}$ David E Simcock, ${ }^{6}$ Benjamin Turner ${ }^{7}$

'Department of Respiratory Medicine, Royal London Hospital, London, UK;

${ }^{2}$ Department of Neurology, University College London Hospital, London, UK;

${ }^{3}$ Department of Microbiology, Royal London Hospital, London, UK; ${ }^{4}$ Department of Histopathology, Royal London Hospital, London, UK; ${ }^{5}$ Department of Microbiology, Royal London Hospital, London, UK; ${ }^{6}$ Department of Respiratory Medicine, Royal London Hospital, London, UK; ' Department of Neurology, Royal London Hospital, London, UK

Correspondence to Dr Paul E Pfeffer, c/o Dr David Simcock, Department of Respiratory Medicine, Royal London Hospital, London E1 1BB, UK; paulpfeffer@hotmail.com

Competing interests None.

Patient consent Obtained.

Provenance and peer review Not commissioned; externally peer reviewed.

Published Online First 1 October 2010

Thorax 2010;65:1066. doi:10.1136/thx.2010.140350
Figure 1 Cross-section $(1.5 \mathrm{~mm})$ CT chest images showing pulmonary nodules.
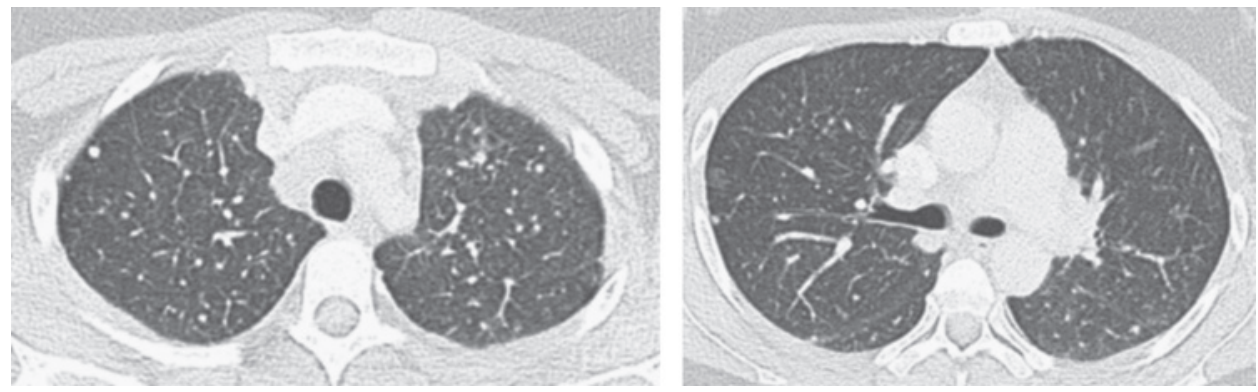